\title{
Electrochemical quantification of iodide ions in synthetic urine using silver nanoparticles: a proof-of-concept
}

\author{
Her Shuang Toh, Kristina Tschulik, Christopher Batchelor-McAuley and Richard G. Compton* \\ Received (in $X X X, X X X) X t h X X X X X X X X X 20 X X$, Accepted Xth $X X X X X X X X X 20 X X$ \\ ${ }_{5}$ DOI: 10.1039/b000000x
}

Typical urinary iodide concentrations range from $0.3 \mu \mathrm{M}$ to $6.0 \mu \mathrm{M}$. The conventional analytical method is based on the Sandell-Kolthoff reaction. It involves the toxic reagent, arsenic acid, and a waiting time of 30 minutes for the iodide ions to reduce the cerium(IV) ions. In the presented work, an alternative fast electrochemical method based on a silver nanoparticle modified electrode is proposed. Cyclic

10 voltammetry was performed with a freshly modified electrode in presence of iodide ions and the voltammetric peaks corresponding to the oxidation of silver to silver iodide and the reverse reaction were recorded. The peak height of the reduction signal of silver iodide was used to plot a calibration line for the iodide ions. Two calibration plots for the iodide ions were obtained, one in $0.1 \mathrm{M}$ sodium nitrate (a chloride-ion free environment to circumvent any interference from the other halides) and another in 15 synthetic urine (which contains $0.2 \mathrm{M} \mathrm{KCl}$ ). In both of the calibration plots, linear relationships were found between the reduction peak height and the iodide ion concentration of $0.3 \mu \mathrm{M}$ to $6.0 \mu \mathrm{M}$. A slope of $1.46 \times 10^{-2} \mathrm{~A} / \mathrm{M}$ and a $\mathrm{R}^{2}$ value of 0.999 were obtained for the iodide detection in sodium nitrate. For the synthetic urine experiments, a slope of $3.58 \times 10^{-3} \mathrm{~A} / \mathrm{M}$ and a $\mathrm{R}^{2}$ value of 0.942 were measured. A robust iodide sensor with the potential to be developed into a point-of-care system has been validated.

\section{${ }_{20} 1$ Introduction}

An iodine deficiency can cause goitre, hyperthyroidism, hypothyroidism and intellectual disability. ${ }^{1-6}$ The most conventional test is an absorbance based urinary iodine analysis because $90 \%$ of iodine is excreted through urine. ${ }^{7}$ A spot urine 25 test (collection of a single sample) or a 24 hour urine sample collection can be used for sample collection. ${ }^{1}$ The SandellKolthoff reaction, involving iodide ions catalysing the reduction of yellow cerium (IV) ions to the colourless form of cerium (III), is used to determine the iodide ion concentration in urine. ${ }^{8}$ After 30 the urine sample is collected, ammonium persulfate is added to reduce iodine to iodide ions. ${ }^{9}$ Then, arsenic acid and cerium ammonium sulphate is added to the sample. The absorbance signal at $420 \mathrm{~nm}$ corresponding to the cerium(IV) ion concentration is measured after 30 minutes and then the number 35 is converted into the urinary iodine concentration. ${ }^{10}$ The current test requires a toxic, carcinogenic reagent (arsenic acid, $\mathrm{H}_{3} \mathrm{AsO}_{3}$ ) and laboratory handling for synthesis, storage and disposal of the reagents. A simpler and non-toxic approach to iodide ion determination is developed in this paper.

40 In this work, we provide a proof-of-concept for an alternative, simple and robust electrochemical method capable of detecting iodide ion levels in synthetic urine. The targeted iodide concentration range is geared to match the World Health Organisation guidelines in Table $1 .^{10}$ Thus, the biological relevant ${ }_{45}$ iodide concentration in this paper is $0.3 \mu \mathrm{M}$ to $6.0 \mu \mathrm{M}$. The main

\begin{tabular}{|c|c|c|}
\hline $\begin{array}{c}\text { Median Urinary } \\
\text { Iodine }(\mu \mathrm{g} / \mathrm{L})\end{array}$ & $\begin{array}{c}\text { Corresponding Iodide } \\
\text { Concentraton }(\mu \mathrm{M})\end{array}$ & Iodine Status \\
\hline$<20$ & $<0.32$ & Severe deficiency \\
\hline $20-49$ & $0.32-0.77$ & Moderate deficiency \\
\hline $50-99$ & $0.79-1.56$ & Mild deficiency \\
\hline $100-199$ & $1.58-3.14$ & Optimal \\
\hline $200-299$ & $3.15-4.71$ & More than adequate \\
\hline$\geq 300$ & $\geq 4.73$ & Excess \\
\hline
\end{tabular}

Table 1. Urinary iodine guidelines for assessing individuals $\geq 6$ years old. $^{10}$

advantages of the proposed method are that the chemicals required in this method are non-toxic and the sample can be 50 processed within a few minutes. The concept behind this present method is the ready formation of silver iodide by oxidation of silver in presence of iodide ions. The iodide sensor is illustrated as a schematic diagram in Figure 1. The test proposed works as follows, a clean glassy carbon electrode was drop cast with silver 55 nanoparticles and dried under nitrogen atmosphere. The modified electrode was exposed to electrolyte spiked with iodide ions and cyclic voltammetry was performed. In the absence of iodide ions, no signal was observed. In the presence of iodide ions, two signals were recorded. Peak I corresponded to the oxidation of 60 silver to silver iodide. The reduction signal of silver iodide is represented by peak II and its peak height was used to derive a calibration curve for the iodide concentration. From there, the unknown iodide concentrations in urine can be determined. In the present method, it is crucial silver nanoparticles are used instead 


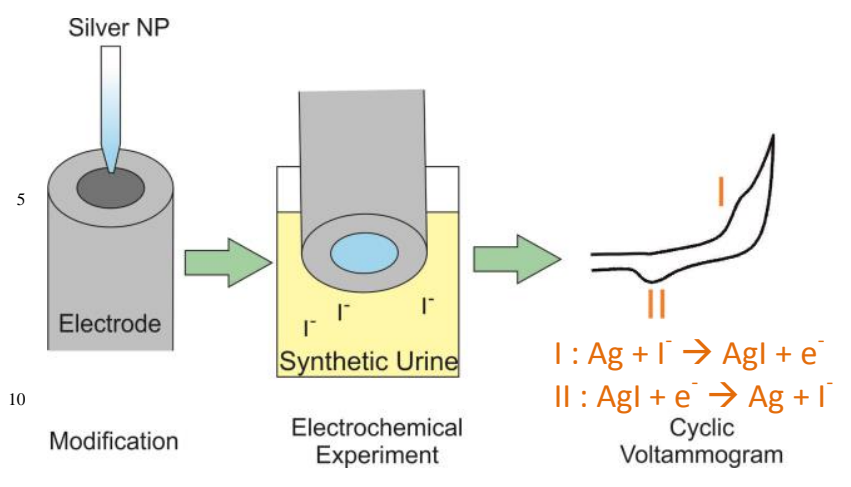

Figure 1. Schematic diagram of silver nanoparticle based iodide sensor. of a bulk silver electrode. The large surface area present allows 15 the pre-concentration of silver iodide on the surface. Using the linear relationship between the reduction peak height and iodide concentration, this system holds great potential for further development into a point-of-care system for urinary iodine detection.

\section{${ }_{20} 2$ Experimental}

\subsection{Chemicals}

Sodium nitrate $\left(>99.5 \%, \mathrm{NaNO}_{3}\right.$ ) was supplied from Fisons Scientific Equipment, Loughborough, UK. Trisodium citrate (> $99 \%, \mathrm{Na}_{3} \mathrm{C}_{6} \mathrm{H}_{5} \mathrm{O}_{7}$ ) was obtained from BDH Laboratory Supplies, 25 Poole, UK. Potassium iodide $(\geq 99.5 \%, \mathrm{KI})$, silver nitrate (> 99\%, $\left.\mathrm{AgNO}_{3}\right)$, sodium borohydride $\left(99 \%, \mathrm{NaBH}_{4}\right)$ were supplied from Sigma-Aldrich, Dorset, UK. Concentrated nitric acid $(>70 \%$, $\left.\mathrm{HNO}_{3}\right)$ and concentrated hydrochloric acid $(37 \%, \mathrm{HCl})$ were obtained from Fisher Scientific, Loughborough, UK. Synthetic 30 urine was obtained from Synthetic Urine e.k., Eberdingen, Germany and used as received. The synthetic urine received adheres to the standards of DIN EN 1616. It contains around 0.2 $\mathrm{M}$ of chloride ions and no iodide ions. All solutions were made with ultrapure water from Millipore with resistivity not less than $3518.2 \mathrm{M} \Omega . \mathrm{cm}$ at $298 \mathrm{~K}$.

\subsection{Voltammetry}

All electrochemical experiments were performed with a three electrode system in a Faraday cage thermostated at $25 \pm 1{ }^{\circ} \mathrm{C}$. A $\mu$ Autolab II from Metrohm-Autolab BV, Utrecht, Netherlands 40 was used as the potentiostat. The working electrode was a glassy carbon electrode of $3.0 \mathrm{~mm}$ diameter from $\mathrm{CH}$ instruments, Austin, US. The electrode was polished to a mirror finish on diamond sprays from Kemet, Kent, UK in the sequence of size $3.0 \mu \mathrm{m}, 1.0 \mu \mathrm{m}$ and $0.1 \mu \mathrm{m}$. For chloride-free experiments, a 45 standard MSE (mercury/mercurous sulphate electrode $\left[\mathrm{Hg} / \mathrm{Hg}_{2} \mathrm{SO}_{4}, \mathrm{~K}_{2} \mathrm{SO}_{4}\right.$ (saturated) $],+0.62 \mathrm{~V}$ vs standard hydrogen electrode) obtained from BASi, West Lafayette, USA was used as a reference electrode. ${ }^{11}$ For the synthetic urine experiments, a SCE (saturated calomel electrode $\left[\mathrm{Hg} / \mathrm{Hg}_{2} \mathrm{Cl}_{2}, \mathrm{KCl}\right.$ (saturated)], + ${ }_{50} 0.24 \mathrm{~V}$ vs standard hydrogen electrode) obtained from Hach Lange, Salford, UK was used as a reference electrode. ${ }^{11} \mathrm{~A}$ platinum mesh was used as a counter electrode. All solutions were thoroughly degassed with nitrogen prior to the experiments.

\subsection{Nanoparticle synthesis and characterisation}

${ }_{55}$ The silver nanoparticles used are capped with citrate and synthesized using the method developed by Wan et al. ${ }^{12}$ They have been fully characterised in a previous paper as Batch II by Lees et al. ${ }^{13}$ The silver nanoparticle suspension has a total silver concentration of $3.1 \mathrm{mM}$ and they were sized by scanning 60 electron microscope to be $13.6 \pm 3.7 \mathrm{~nm}$ in radii.

\section{'2.4 Detection procedures}

A freshly prepared silver nanoparticle modified electrode was used for electrochemical experiments. The modification was performed by diluting the synthesized silver nanoparticle 65 suspension with ultrapure water by a factor of 100 to ensure the diluted suspension contained $31 \mu \mathrm{M}$ of silver. $3 \mu \mathrm{L}$ of the diluted nanoparticle suspension was drop cast onto a glassy carbon electrode and dried under a nitrogen flow. The dried electrode was used immediately. The concentrations of potassium iodide $70(\mathrm{KI})$ tested were $0.3 \mu \mathrm{M}, 0.8 \mu \mathrm{M}, 1.6 \mu \mathrm{M}, 3.2 \mu \mathrm{M}, 4.7 \mu \mathrm{M}$ and $6.0 \mu \mathrm{M}$. The solutions were all degassed with nitrogen before the start of the experiments.

\subsubsection{Detection procedure in sodium nitrate}

$0.3 \mu \mathrm{M}$ to $6.0 \mu \mathrm{M}$ of $\mathrm{KI}$ were made up by dissolving different 75 amounts of $\mathrm{KI}$ in $0.1 \mathrm{M}$ sodium nitrate. The scan rate was $10 \mathrm{mV} \mathrm{s}^{-1}$ and a MSE was used as a reference electrode to maintain the chloride-free environment to circumvent the possible interferences from other halides. The voltammetric scan started by scanning oxidatively from $-0.6 \mathrm{~V}$ to $-0.2 \mathrm{~V}$ and back 80 to $-0.7 \mathrm{~V}$ vs MSE.

\subsubsection{Detection procedure in synthetic urine}

Different amounts of KI were dissolved in synthetic urine to make up the range from $0.3 \mu \mathrm{M}$ to $6.0 \mu \mathrm{M}$. They were used as the electrolyte for the electrochemical experiments. A SCE reference 85 electrode was used. The cyclic voltammetric scan started from $0.2 \mathrm{~V}$ to $+0.045 \mathrm{~V}$ and then back to $-0.3 \mathrm{~V}$ vs SCE at $5 \mathrm{mV} \mathrm{s}^{-1}$.

\subsubsection{Detection procedure for standard addition}

A synthetic urine sample containing $0.2 \mu \mathrm{M}$ of $\mathrm{KI}$ was separated into portions and an additional $0.5 \mu \mathrm{M}, 1.0 \mu \mathrm{M}$ and $1.5 \mu \mathrm{M}$ of $\mathrm{KI}$

90 were spiked in the separate sample portions. Then, a freshly prepared silver nanoparticle modified electrode was used to perform the cyclic voltammetry which started from $-0.2 \mathrm{~V}$ to $+0.045 \mathrm{~V}$ and back to $-0.3 \mathrm{~V}$ vs SCE at $5 \mathrm{mV} \mathrm{s}^{-1}$.'

\section{Results and Discussion}

${ }_{95}$ Herein, the detection of iodide ions using a silver nanoparticle modified electrode is reported. The diluted silver nanoparticle suspension was drop cast onto a polished glassy carbon electrode, dried under a nitrogen atmosphere and used to record a cyclic voltammogram in a degassed electrolyte containing iodide ions. 100 An oxidative peak was observed for the oxidation of silver to silver iodide and a reductive peak was observed for the reverse reaction. The peak height of the latter was used to establish a calibration curve to measure the iodide concentration.

One important factor to be considered in this system is the 105 presence of chloride ions. The formation of silver iodide is thermodynamically favoured compared to the formation of silver(I) ions. Therefore, the oxidation to silver iodide occurs at a lower potential compared to the oxidation to silver(I) ions. In previous literature, this concept has also been applied to chloride 110 detection. ${ }^{14,} 15$ The thermodynamically favoured formation of silver chloride also decreases the oxidation peak potential. 
Therefore, the iodide sensor was first tested in a chloride-free environment to prevent the possible interference from chloride ions (Section 3.1). Nonetheless, the ultimate aim was to detect iodide ions in synthetic urine and synthetic urine contains $0.2 \mathrm{M}$ 5 of chloride ions. The oxidation potentials for the formation of silver chloride and silver iodide both depend greatly upon of the halide ion concentrations. In the presence of $0.2 \mathrm{M}$ of chloride ions, the oxidation signal for the formation of silver iodide, at the medically relevant iodide concentrations, occurs at a lower ${ }_{10}$ potential than the silver-silver chloride redox couple. Thus, the potential window was optimised to stop before the bulk formation of silver chloride (Section 3.2). Thus, it is possible to detect the iodide ions despite the presence of chloride ions. In Section 3.3, the measurement of a low iodide concentration was attempted 15 through the standard addition method.

\subsection{Iodide Calibration in Sodium Nitrate}

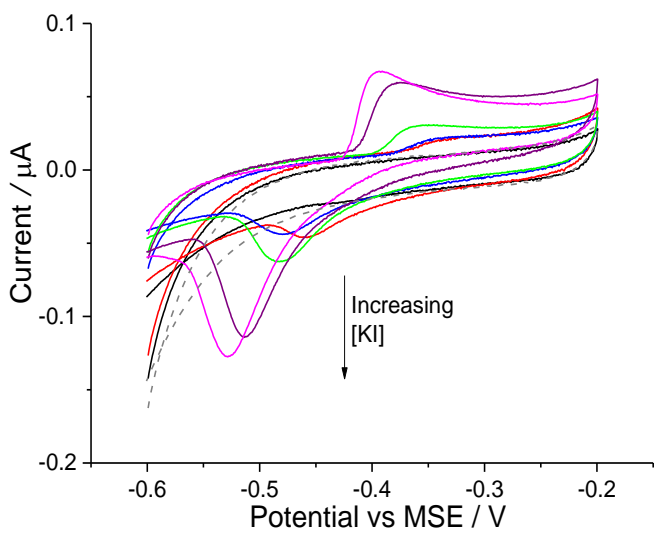

Figure 2. The oxidation of silver nanoparticles on a glassy carbon electrode in $0.1 \mathrm{M}$ sodium nitrate spiked with different concentrations of potassium iodide (KI) at a scan rate of $10 \mathrm{mV} \mathrm{s}-1$. Grey dashed: No Kl; 20 Black: $0.3 \mu \mathrm{M} \mathrm{KI}$; Red: $0.8 \mu \mathrm{M} \mathrm{Kl}$; Blue: $1.6 \mu \mathrm{M} \mathrm{KI}$; Green: $3.2 \mu \mathrm{M} \mathrm{KI}$; Purple: $4.7 \mu \mathrm{M} \mathrm{KI}$; Pink: $6.0 \mu \mathrm{M} \mathrm{KI}$.

Figure 2 shows the close up of the voltammograms for silver nanoparticle oxidation in the presence of different concentrations of KI. In the absence of KI (dashed grey line in Figure 2), no 25 voltammetric peak was observed. In the presence of KI, an anodic peak occurred between $-0.3 \mathrm{~V}$ to $-0.4 \mathrm{~V}$ and it corresponds to the oxidation of silver to silver iodide.

$$
\mathrm{Ag}+\mathrm{I}^{-} \rightarrow \mathrm{AgI}+\mathrm{e}^{-}
$$

A reduction signal occurred between $-0.4 \mathrm{~V}$ to $-0.55 \mathrm{~V}$ in the 30 presence of $\mathrm{KI}$ and it is accounted by the reduction of the previously formed silver iodide to silver.

$$
\mathrm{AgI}+\mathrm{e}^{-} \rightarrow \mathrm{Ag}+\mathrm{I}^{-}
$$

Both the oxidation and reduction signal correlate linearly with the concentration of KI present. The reduction signal has a larger 35 peak height as it is a surface bound wave and reflects the quantity of silver iodide present. Thus, the peak height of the reduction signal was plotted against the concentration of KI in Figure 3 to achieve a greater sensitivity. The experiment at each individual KI concentration was repeated for at least three times with a 40 freshly prepared silver nanoparticle modified electrode (Section 2.4) to determine a standard deviation. A linear relationship was Figure 3. Calibration plot of reduction peak height against the

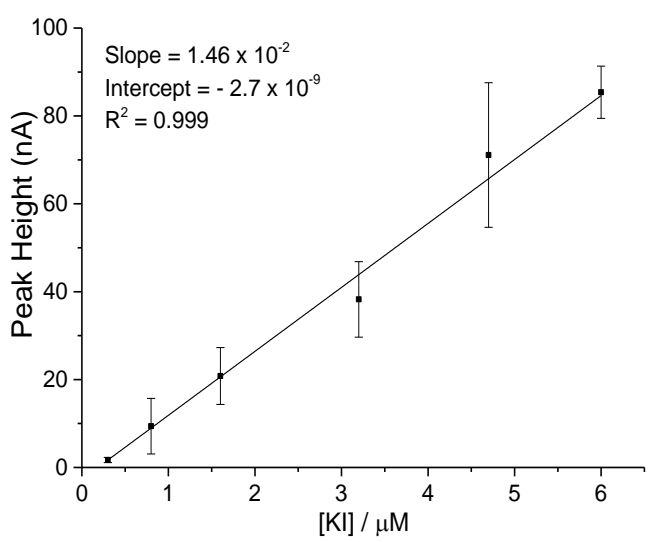

concentration of potassium iodide $(\mathrm{KI})$ in $0.1 \mathrm{M}$ sodium nitrate. Each data point consists of a minimum of 3 repeats.

45 found, giving a slope of $1.46 \times 10^{-2} \mathrm{~A} / \mathrm{M}$ and $\mathrm{a} \mathrm{R}^{2}$ (coefficient of determination) value of 0.999 . The $R^{2}$ is a value between 0 and 1 and a high number indicates a good correlation between the data points and the trend. The linear relationship is determined to be:

$$
\left[\mathrm{I}^{-}\right]=\left(\mathrm{m}_{1}\right)\left(i_{\mathrm{p}}\right)+\mathrm{C}_{1}
$$

50 where $\left[\mathrm{I}^{-}\right]$is concentration of iodide ions $\left(\mathrm{mol} \mathrm{L} \mathrm{L}^{-1}\right), \mathrm{m}_{1}$ is the gradient of $1.46 \times 10^{-2} \mathrm{~A} \mathrm{~mol}^{-1} \mathrm{~L}, \mathrm{i}_{\mathrm{p}}$ is the peak height $(\mathrm{A})$ and $\mathrm{C}_{1}$ is the intercept of $-2.7 \times 10^{-9} \mathrm{~mol} \mathrm{~L}^{-1}$.

\subsection{Iodide Calibration in Synthetic Urine}

The analytical system introduced in Section 3.1 was further 55 optimised to determine the iodide ions concentration in synthetic urine. The synthetic urine supplied contains $0.2 \mathrm{M}$ of chloride ions. The synthetic urine sample did not contain iodide ions and iodine. Hence, the same range of KI as in Section $3.1(0.3 \mu \mathrm{M}$ to $6.0 \mu \mathrm{M})$ was spiked into the synthetic urine. No additional 60 supporting electrolyte was added. Due to the presence of chloride ions, the reference electrode was changed from a MSE to a SCE. It is known that silver nanoparticles oxidise to silver chloride in the presence of chloride ions. ${ }^{14,15}$ The large signal which arises from the formation of silver chloride might dominate the signal of 65 interest. The solubility product of silver iodide and silver chloride at $25{ }^{\circ} \mathrm{C}$ are $1.5 \times 10^{-16} \mathrm{~mol}^{2} \mathrm{dm}^{-6}$ and $1.6 \times 10^{-10} \mathrm{~mol}^{2} \mathrm{dm}^{-6}$ respectively. ${ }^{11}$ The stronger affinity of iodide to silver causes the oxidation of silver to silver iodide to occur at a lower potential compared to the oxidation of silver chloride. Hence, cyclic 70 voltammetry was performed by sweeping oxidatively from -0.2 $\mathrm{V}$ to $+0.045 \mathrm{~V}$ and then back to $-0.3 \mathrm{~V}$ vs SCE to limit the potential window so as to exclude potential at which silver chloride is formed. ${ }^{\mathrm{i}}$ No signal of interest was detected for $0.3 \mu \mathrm{M}$ KI when the system was applied in synthetic urine. Neither the 75 oxidation of silver to silver iodide nor the reduction of silver iodide was seen. Only the initial onset of the oxidation of silver to silver chloride was observed. Therefore, the scan rate was reduced from $10 \mathrm{mV} \mathrm{s}^{-1}$ to $5 \mathrm{mV} \mathrm{s}^{-1}$ to ensure that the signal of interest was observed across the entire concentration range of 80 interest. The optimised detection procedure is summarised in

\footnotetext{
i The corresponding potential window versus MSE is $-0.58 \mathrm{~V}$ to -0.335 $\mathrm{V}$ to $-0.68 \mathrm{~V}$.
} 


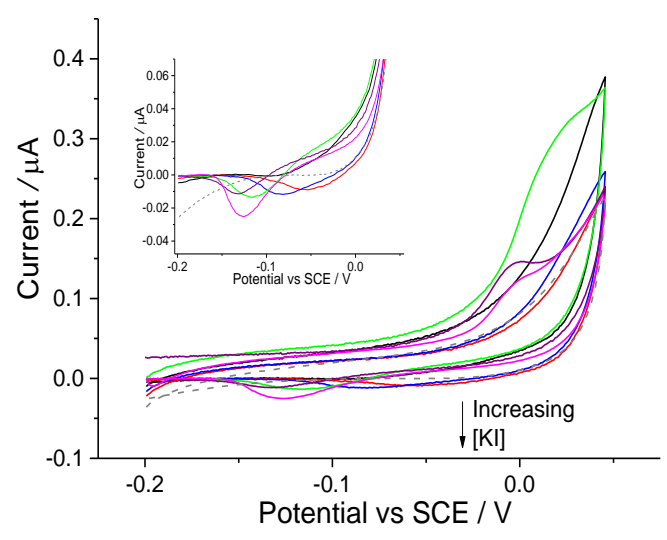

Figure 4. The oxidation of silver nanoparticles on a glassy carbon electrode in synthetic urine spiked with different concentrations of potassium iodide (KI) at a scan rate of $5 \mathrm{mV} \mathrm{s}-1$. Grey dashed: No Kl; Black: $0.3 \mu \mathrm{M} \mathrm{Kl}$; Red: $0.8 \mu \mathrm{M} \mathrm{Kl}$; Blue: $1.6 \mu \mathrm{M} \mathrm{Kl}$; Green: $3.2 \mu \mathrm{M} \mathrm{Kl}$; 5 Purple: $4.7 \mu \mathrm{M} \mathrm{KI}$; Pink: $6 \mu \mathrm{M} \mathrm{KI}$. Inlay: a further close up of the reduction signal of interest at different concentrations of KI.

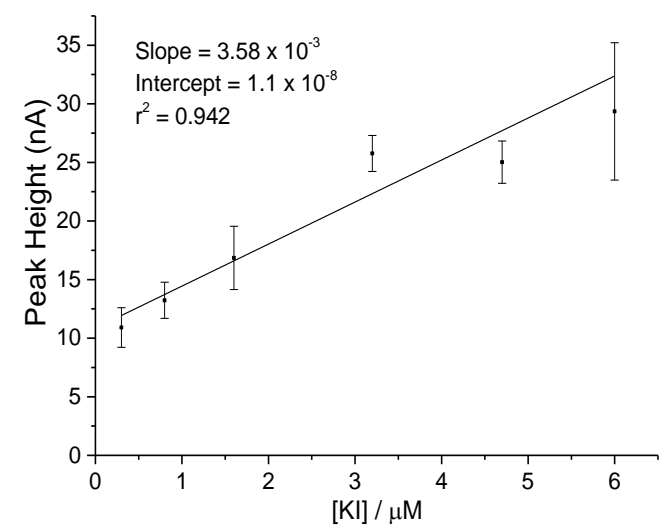

Figure 5. Calibration plot of peak height against the concentration of potassium iodide in synthetic urine. Each data point consists of a 10 minimum of 3 repeats.

Section 2.4 and Section 2.4.2. Performing the experiments with the optimised system, the close-up of the voltammograms recorded with different concentrations of iodide ions are depicted in Figure 4. It can be seen that the oxidation of silver to silver 15 iodide at $-0.02 \mathrm{~V}$ to $+0.045 \mathrm{~V}$ vs SCE occurred on the steep slope which was the start of a large oxidation signal of silver to silver chloride. The peak of interest now occurred as a reductive signal between $0 \mathrm{~V}$ to $\quad-0.15 \mathrm{~V}$ vs SCE. The peak height of the signal was plotted against the concentration of KI in Figure 5 and 20 a linear calibration curve was obtained. A slope of $3.58 \times 10^{-3} \mathrm{~A} /$ $\mathrm{M}$ was measured and a $\mathrm{R}^{2}$ value of 0.942 was obtained. The linear relationship is determined to be:

$$
\left[I^{-}\right]=\left(m_{2}\right)\left(i_{p}\right)+C_{2}
$$

where $\left[\mathrm{I}^{-}\right]$is the concentration of iodide ions $\left(\mathrm{mol} \mathrm{L}^{-1}\right), \mathrm{m}_{2}$ is the 25 gradient of $3.58 \times 10^{-3} \mathrm{~A} \mathrm{~mol}^{-1} \mathrm{~L}, \mathrm{i}_{\mathrm{p}}$ is the peak height (A) and $\mathrm{C}_{2}$ is the intercept of $1.1 \times 10^{-8} \mathrm{~mol} \mathrm{~L}^{-1}$. This showed that silver nanoparticles can be employed to detect iodide ions in synthetic urine.

\subsection{Standard Addition Method to Measure the Iodide ${ }_{30}$ Concentration}

To further validate this method, the standard addition method was used on a sample of synthetic urine containing (spiked with) 0.2 $\mu \mathrm{M}$ of KI. This particularly low concentration was chosen as it is challenging to measure very low iodide concentrations. The

35 chosen method of standard addition refers to the addition of known concentrations of analyte to deduce the unknown amount of analyte present in the sample. ${ }^{16}$ Here, a synthetic urine sample containing $0.2 \mu \mathrm{M}$ of $\mathrm{KI}$ was separated into portions and an additional $0.5 \mu \mathrm{M}, 1.0 \mu \mathrm{M}$ and $1.5 \mu \mathrm{M}$ of $\mathrm{KI}$ were spiked in the 40 separate sample portions. The optimised system in Section 3.2 was used to measure the concentration of iodide ions in the spiked samples. The iodide concentration measured was derived from the calibration equation (4) obtained in Section 3.2. Instead of the traditional standard addition plot where the measured 45 signal is plotted against the KI concentration added, in Figure 6, the concentration measured was plotted against added concentration. This is because the calibration plot in Figure 5 has a non-zero intercept and this is attributed to the underlying electrochemical reduction of silver chloride formed. It is seen that 50 the results of this standard addition test deemed the urine sample to contain $0.29 \pm 0.11 \mu \mathrm{M}$. This is a good estimate as the actual $\mathrm{KI}$ concentration in the urine sample is $0.2 \mu \mathrm{M}$.

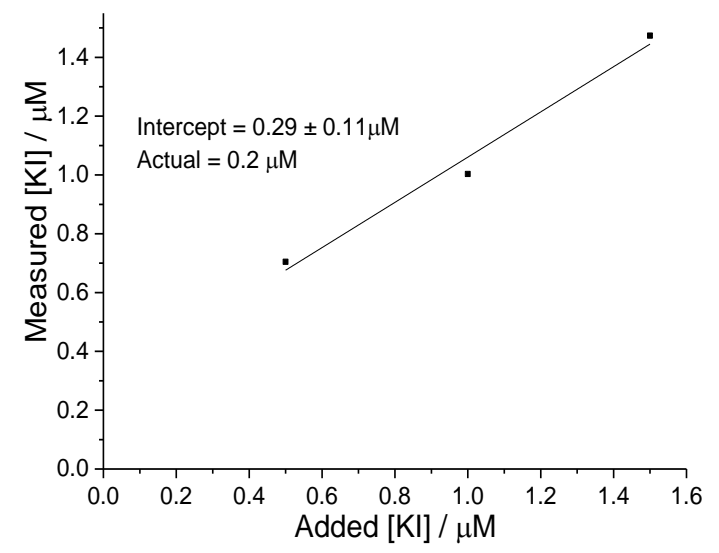

Figure 6. Standard addition plot to determine the concentration of 55 iodide in synthetic urine. Calculations are based on the calibration graph in synthetic urine in Equation 4.

\section{Conclusions}

The measurement of urinary iodine concentration is a key indicator of the health of the thyroid. Both excess and insufficient ${ }_{60}$ iodine in the body can lead to swelling of the thyroid. Hyperactivity or hypoactivity of the gland is also possible. Although the urine samples are easily obtainable, the standard assessment based on Sandell-Kolthoff reaction requires a carcinogenic reagent and a thirty minutes incubation before an ${ }_{65} \mathrm{UV}$-vis absorbance measurement. In this work, the silver nanoparticle modified electrode used is capable of detecting the biologically relevant iodide range of $0.3 \mu \mathrm{M}$ to $6.0 \mu \mathrm{M}$ in the 
synthetic urine. Moreover, the sample processing time is reduced to a few minutes. In samples with very low iodide concentration, standard addition can be performed to determine the urinary iodide concentration. Therefore, this system is capable of 5 detecting the iodide concentration in urine. To utilise this sensor for urinary iodine test, a reducing agent can be added to convert the iodine in urine. This in turn allows to the distinguishing of the various iodine statuses (deficiency, optimal or excess) in individuals in a reliable and non-toxic manner.

\section{${ }_{10} 5$ Acknowledgements}

HST is supported by the National Research Foundation Singapore under its National Research Foundation (NRF) Environmental and Water Technologies (EWT) PhD Scholarship Programme and administered by the Environment and Water Industry 15 Programme Office (EWI). KT is funded by a Marie Curie Intra European Fellowship. The research leading to these results has received partial funding from the European Research Council under the European Union's Seventh Framework Programme (FP/2007-2013)/ERC Grant Agreement no. [320403].

\section{${ }_{20}$ Authors Information}

Department of Chemistry, Physical and Theoretical Chemistry Laboratory, Oxford University, South Parks Road, Oxford, OX1 3QZ, United Kingdom

*Correspondence E-mail: Richard.Compton@chem.ox.ac.uk

\section{${ }_{25} 6$ References}

1. M. B. Zimmermann, Endocr Rev, 2009, 30, 376-408.

2. G. M. Abrams and P. R. Larsen, J Clin Invest, 1973, 52, 25222531.

3. F. Delange, M. Camus and A. M. Ermans, J Clin Endocr

3. Metab, 1972, 34, 891-895.

4. P. Laurberg, C. Cerqueira, L. Ovesen, L. B. Rasmussen, H. Perrild, S. Andersen, I. B. Pedersen and A. Carle, Clin Endocr Metab, 2010, 24, 13-27.

5. E. Nyström, G. E. B. Berg, S. K. G. Jansson, O. Tørring and S.

35 V. Valdemarsson, Thyroid Disease in Adults, Springer, Berlin, Germany, 2011.

6. F. Delange, Postgrad Med J, 2001, 77, 217-220.

7. BCC Research. Nanotechnology: A Realistic Market Assessment. Feb 2014 http://www.bccresearch.com/market-

$40 \quad$ research/nanotechnology/nanotechnology-market-

applications-products-nan031e.html.

8. E. B. Sandell and I. M. Kolthoff, Mikrochim Acta, 1937, 1, 9 25.

9. S. Pino, S.-L. fang and L. E. Braverman, Clin Chem, 1996, 42, 45 239-243.

10. World Health Organisation, Assessment of iodine deficiency disorders and monitoring their elimination: a guide for programme managers. 3rd edition. Feb 2014, http://www.who.int/nutrition/publications/micronutrients/io 50 dine_deficiency/9789241595827/en/.

11. W. M. Haynes, D. R. Lide and T. J. Bruno, CRC Handbook of Chemistry and Physics 2012-2013, Taylor \& Francis Group, Florida, 93rd edn., 2012.

12. Y. Wan, Z. Guo, X. Jiang, K. Fang, X. Lu, Y. Zhang and N.

$55 \quad \mathrm{Gu}, \mathrm{J}$ Colloid Interface Sci, 2013, 394, 263-268.

13. J. C. Lees, J. Ellison, C. Batchelor-McAuley, K. Tschulik, C. Damm, D. Omanovic and R. G. Compton, Chemphyschem, 2013, 14, 3895-3897.

14. H. S. Toh, C. Batchelor-McAuley, K. Tschulik and R. G. 60 Compton, Analyst, 2013, 138, 4292-4297.
15. C. Villagran, C. E. Banks, C. Hardacre and R. G. Compton, Anal Chem, 2004, 76, 1998-2003.

16. D. A. Skoog, D. M. West, F. J. Holler and S. R. Crouch, Fundamentals of Analytical Chemistry, Thompson

65 Brooks/Cole, Belmont, USA, 2004. 